\title{
Prediction of rating from comments based on information retrieval and sentiment analysis
}

\begin{abstract}
The number of users of an on-line shopping websites is continuously increasing. Such website often provides facility for the users to give comments and ratings to the products being sold on the websites. This information can be useful as the recommendation for other users in making their purchase decision. This paper investigates the problem of predicting rating based on users' comments. A classifier based on information retrieval model is proposed for the prediction. In addition, the effect of integrating sentiment analysis for the rating prediction is also investigated. Based on the results, an improvement in prediction performance can be expected with sentiment analysis where an increase of 54\% is achieved.
\end{abstract}

Keyword: Comments; Information retrieval; Rating; Sentiment analysis; Vector space model 